\title{
Vector bundles over Dold manifolds
}

by

R. E. Stong (Charlottesville, VA)

Abstract. This paper determines the possible Stiefel-Whitney classes for vector bundles over Dold manifolds.

1. Introduction. The Dold manifolds

$$
P(m, n)=\frac{S^{m} \times \mathbb{C} P^{n}}{-1 \times(\text { conjugation })}
$$

were introduced by Dold [2] in order to find odd-dimensional generators for the unoriented cobordism ring. They are finite-dimensional approximations to the classifying space $\mathrm{BO}_{2}=P(\infty, \infty)$ for real 2-plane bundles.

The objective of this paper is to determine the possible Stiefel-Whitney classes of the vector bundles over $P(m, n)$. Knowing these classes is crucial for cobordism calculations involving the Dold manifolds; particularly in studying involutions for which some fixed component is a Dold manifold.

The mod 2 cohomology of the Dold manifold is given by

$$
H^{*}\left(P(m, n) ; Z_{2}\right)=Z_{2}[c, d] /\left(c^{m+1}=d^{n+1}=0\right),
$$

where $c \in H^{1}\left(P(m, n) ; Z_{2}\right)$ and $d \in H^{2}\left(P(m, n) ; Z_{2}\right)$. The action of the Steenrod algebra is completely determined by knowing that $\mathrm{Sq}^{1} d=c d$. Over $P(m, n)$ one has a real line bundle $\ell$ with $w(\ell)=1+c$ and a real 2-plane bundle $\eta$ with $w(\eta)=1+c+d$. Thus, there are vector bundles over $P(m, n)$ with Stiefel-Whitney classes of the form $(1+c)^{a}(1+c+d)^{b}$.

The KO-theory of $P(m, n)$ was determined by Fujii and Yasui [3] and by Ucci. (Ucci's paper [4] gives a very partial description and promises the complete calculation, but as far as I know that has never appeared.) The description of $\operatorname{KO}(P(m, n))$ is quite complicated, and does not describe the Stiefel-Whitney classes.

The main result of this paper is

2000 Mathematics Subject Classification: Primary 55R40. 
Proposition. There are vector bundles over $P(m, n)$ with StiefelWhitney classes

(1) $1+c+\left(d+c^{2}\right)$, for $m=2, n \geq 1$,

(2) $\left(1+c+\left(d+c^{2}\right)\right)^{2}$, for $m=4$ or $5, n \geq 2$,

(3) $\left(1+c+\left(d+c^{2}\right)\right)^{2}(1+c+d)+c^{6}$, for $m=6, n \geq 1$, and

(4) $1+c^{2} d^{3}$, for $m=2, n=3$.

The Stiefel-Whitney class of every vector bundle is a product of these classes and the classes $1+c$ and $1+c+d$.

Notes. (1) The squares of each of these classes are of the form $(1+c+d)^{b}$, so at most a single factor of each of these is needed.

(2) For $m=2$ and $n=3$, there are two classes in the list. In all other cases, there is only one exotic class.

2. The bundles. From the $\mathrm{Wu}$ formulae

$$
\mathrm{Sq}^{i} w_{j}=\sum_{t=0}^{i}\left(\begin{array}{c}
j-i-1+t \\
t
\end{array}\right) w_{i-t} w_{j+t} \quad \text { for } i<j
$$

for Steenrod operations on Stiefel-Whitney classes, it follows that for any vector bundle $\xi$ there is an $s$ with

$$
w(\xi)=1+w_{2^{s}}(\xi)+\text { terms of larger dimension, }
$$

and that

$$
\mathrm{Sq}^{i} w_{2^{s}}(\xi)=0 \quad \text { for } 0<i<2^{s-1} .
$$

If one has two vector bundles $\xi, \xi^{\prime}$ with

$$
w(\xi)=1+w_{2^{s}}(\xi)+\ldots, \quad w\left(\xi^{\prime}\right)=1+w_{2^{s}}\left(\xi^{\prime}\right)+\ldots,
$$

then for the Whitney sum

$$
w\left(\xi \oplus \xi^{\prime}\right)=w(\xi) \cdot w\left(\xi^{\prime}\right)=1+\left(w_{2^{s}}(\xi)+w_{2^{s}}\left(\xi^{\prime}\right)\right)+\ldots
$$

Over $P(m, n)$ one has vector bundles $\ell$ and $\eta-\ell$ with

$$
w(\ell)=1+c, \quad w(\eta-\ell)=\frac{1+c+d}{1+c}=1+d+c d+c^{2} d+\ldots,
$$

and for each $s$,

$$
w\left(2^{s} \ell\right)=1+c^{2^{s}}, \quad w\left(2^{s-1}(\eta-\ell)\right)=1+d^{2^{s-1}}+\ldots
$$

If $\xi$ is a vector bundle over $P(m, n)$ with $w(\xi)$ not of the form $(1+c)^{a}(1+c+d)^{b}$, then by adding copies of $\ell$ and $\eta-\ell$ to $\xi$, one may suppose

$$
w(\xi)=1+w_{2^{s}}(\xi)+\text { higher terms, }
$$

with $2^{s} \geq 4$ where $w_{2^{s}}(\xi)$ is a nonzero sum of terms $c^{2 i} d^{2^{s-1}-i}$ with $0<i$ $<2^{s-1}, 2 i \leq m, 2^{s-1}-i \leq n$. The fact that $2^{s} \geq 4$ is immediate since one 
may cancel $c$ and both $c^{2}$ and $d$ in dimensions 1 and 2. (Note that any vector bundle with Stiefel-Whitney class of this form cannot have Stiefel-Whitney class of the form $(1+c)^{a}(1+c+d)^{b}$.)

If one writes

$$
w_{2^{s}}(\xi)=\sum \alpha_{i} c^{2 i} d^{2^{s-1}-i} \quad\left(0<i<2^{s-1}, 2 i \leq m, 2^{s-1}-i \leq n\right),
$$

then

$$
0=\mathrm{Sq}^{1} w_{2^{s}}(\xi)=\sum \alpha_{i} c^{2 i}\left(2^{s-1}-i\right) c d^{2^{s-1}-i}=\sum i \alpha_{i} c^{2 i+1} d^{2^{s-1}-i} .
$$

First, suppose $m$ is odd. In this case, $2 i \leq m$ implies $2 i+1 \leq m$ so $\mathrm{Sq}^{1} w_{2^{s}}(\xi)=0$ forces all nonzero terms in $w_{2^{s}}(\xi)$ to have $i$ even. Thus $w_{2^{s}}(\xi)=\sum \alpha_{2 j} c^{4 j} d^{2^{s-1}-2 j}$. If $n$ is odd, then $w_{2^{s}}(\xi) \neq 0$ implies $w_{2^{s}}(\xi) \neq 0$ in $P(m, n-1)$, since the powers of $d$ occurring are even, so one may suppose $n$ is even.

For $m \equiv 1,3$, or $7 \bmod 8$, Fujii and Yasui [3, Theorem 5] show that $\operatorname{KO}(P(m, n))$ with $n$ even is generated by $\ell$ and the tensor powers of $\eta$. Now, for tensor powers one has

$$
w(\ell \otimes \ell)=1, \quad w(\ell \otimes \eta)=(1+c)^{2}+c(1+c)+d=1+c+d,
$$

and writing $(1+c+d)=(1+x)(1+y)$ with the splitting principle,

$$
\begin{aligned}
w(\eta \otimes \eta)= & \left((1+x)^{2}+c(1+x)+d\right)\left((1+y)^{2}+c(1+y)+d\right) \\
= & \left(1+c+\left(d+c x+x^{2}\right)\right)\left(1+c+\left(d+c y+y^{2}\right)\right) \\
= & (1+c)^{2}+(1+c)\left(d+c x+x^{2}+d+c y+y^{2}\right) \\
& +\left\{d^{2}+d\left(c x+x^{2}+c y+y^{2}\right)+x y(c+x)(c+y)\right\} \\
= & 1+c^{2}+(1+c)\left(c(x+y)+(x+y)^{2}\right) \\
& +\left\{d^{2}+d\left(c(x+y)+(x+y)^{2}\right)+x y\left(c^{2}+c(x+y)+x y\right)\right\} \\
= & 1+c^{2}+(1+c)\left(c^{2}+c^{2}\right)+\left\{d^{2}+d\left(c^{2}+c^{2}\right)+d\left(c^{2}+c^{2}+d\right)\right\} \\
= & 1+c^{2} \\
= & (1+c)^{2},
\end{aligned}
$$

and the tensor powers contribute no additional Stiefel-Whitney classes. Thus, every vector bundle has class of the form $(1+c)^{a}(1+c+d)^{b}$, for $m \equiv 1,3,7 \bmod 8$.

For $m \equiv 5 \bmod 8$, Fujii and Yasui [3, Theorem 5] show that the restriction homomorphism $\operatorname{KO}(P(m, n)) \leftarrow \operatorname{KO}\left(P\left(m, n^{\prime}\right)\right)$ is always epic for $n \leq n^{\prime}$. Hence, a nonstandard bundle must occur for all $n^{\prime} \geq n$. If $w_{2^{s}}(\xi) \neq 0$, restricting to $P(m-2, n)$ must send $w_{2^{s}}(\xi)$ to zero (for $m-2 \equiv 3 \bmod 8$ ), and so one has

$$
w_{2^{s}}(\xi)=c^{m-1} d^{2^{s-1}-(m-1) / 2} .
$$


Letting $m=8 u+5$ gives

$$
w_{2^{s}}=c^{8 u+4} d^{2^{s-1}-(4 u+2)},
$$

and

$$
\begin{aligned}
\operatorname{Sq} w_{2^{s}}(\xi) & =c^{8 u+4} d^{2^{s-1}-(4 u+2)}(1+c+d)^{2^{s-1}-(4 u+2)} \\
& =c^{8 u+4} d^{2^{s-1}-(4 u+2)}(1+d)^{2^{s-1}-(4 u+2)}
\end{aligned}
$$

since $c^{8 u+4}$ annihilates $c^{2}$. Now $2^{s-1}-(4 u+2)>0$, so $2^{s-1}>2$, so $2^{s-1}$ is divisible by 4 , so

$$
\mathrm{Sq}^{4} w_{2^{s}}(\xi)=c^{8 u+4} d^{2^{s-1}-4 u} \neq 0 .
$$

(Note: $n$ is large.) Since $\mathrm{Sq}^{i} w_{2^{s}}(\xi)=0$ for $0<i<2^{s-1}$, one must have $2^{s-1} \leq 4$, forcing $2^{s-1}=4$, and as $2^{s-1}$ is greater than $4 u+2$, one must have $u=0$. Thus, if a bundle with exotic Stiefel-Whitney class occurs for $m \equiv 5 \bmod 8$, one has $m=5,2^{s}=8$, and $w_{8}(\xi)=c^{4} d^{2}$ (and $n \geq 2$, clearly).

Now suppose $m$ is even. The restriction of $\xi$ to $P(m-1, n)$ must be standard or $m=6,2^{s}=8$, so

$$
w_{2^{s}}(\xi)=\left\{\begin{array}{l}
c^{m} d^{2^{s-1}-m / 2} \\
\alpha c^{6} d+c^{4} d^{2}
\end{array} \quad \text { if } m=6,2^{s}=8 .\right.
$$

For $m=6,2^{s}=8,0=\mathrm{Sq}^{2} w_{8}(\xi)=\alpha c^{6} d^{2}+c^{4}\left(c^{2} d^{2}\right)=(\alpha+1) c^{6} d^{2}$, which forces $\alpha=1$.

Now suppose $w_{2^{s}}(\xi)=c^{m} d^{2^{s-1}-m / 2}, 2^{s-1}-m / 2>0$, so $2^{s}>m$ and $n \geq 2^{s-1}-m / 2$.

For $m \equiv 0 \bmod 4,2^{s-1}-m / 2$ is even and one may restrict to $P\left(m, 2^{s-1}-m / 2\right)$ with $w_{2^{s}}(\xi) \neq 0$. For $m \equiv 0 \bmod 8$, Fujii and Yasui [3] show that $\operatorname{KO}(P(m, n))$ with $n$ even has generators $\ell$ and the tensor powers of $\eta$, so every bundle has class of the form $(1+c)^{a}(1+c+d)^{b}$. For $m \equiv 4 \bmod 8$, the restriction $\mathrm{KO}(P(m, n)) \leftarrow \mathrm{KO}\left(P\left(m, n^{\prime}\right)\right)$ with $n$ and $n^{\prime}$ even and $n^{\prime} \geq n$ is epic, so the exotic bundle would exist for all $n$. For $m=8 u+4, w_{2^{s}}=c^{8 u+4} d^{2^{s-1}-4 u-2}$ and $2^{s-1}>4 u+2$ implies $2^{s-1} \geq 4$ and $2^{s-1}$ is divisible by 4 . Taking $n$ large, we get

$$
\mathrm{Sq}^{4} w_{2^{s}}(\xi)=\mathrm{Sq}^{4}\left(c^{8 u+4} d^{2^{s-1}-4 u-2}\right)=c^{8 u+4} d^{2^{s-1}-4 u} \neq 0,
$$

from which $2^{s-1} \leq 4$. Thus $2^{s-1}=4$ and $4 \geq 2^{s-1}>4 u+2$ forces $u=0$. Thus, if a bundle with exotic Stiefel-Whitney class occurs for $m \equiv 0 \bmod 4$, one has $m=4,2^{s}=8$, and $w_{8}(\xi)=c^{4} d^{2}$ (and $n \geq 2$, clearly).

For $m \equiv 6 \bmod 8$, the restriction $\mathrm{KO}(P(m, n)) \leftarrow \operatorname{KO}\left(P\left(m, n^{\prime}\right)\right)$ is epic for all $n^{\prime} \geq n$. Let $m=8 u+6, n>2^{s-1}-4 u-3$. Then $w_{2^{s}}(\xi)=$ $c^{8 u+6} d^{2^{s-1}-4 u-3}$ gives

$$
\mathrm{Sq}^{2} w_{2^{s}}(\xi)=c^{8 u+6} d^{2^{s-1}-4 u-2} \neq 0,
$$


which is a contradiction unless $2^{s} \leq 4$ and $2 \geq 2^{s-1}>4 u+3$. Thus, the bundle cannot extend to $P\left(m, 2^{s-1}-4 u-2\right)$ to have $w_{2^{s}}(\xi)=c^{8 u+6} d^{2^{s-1}-4 u-3}$. Thus, $m=6, n=1$, and $2^{s}=8$ with $w_{8}(\xi)=c^{6} d$, extending to $m=6$, $n=2$ to have $w_{8}(\xi)=c^{6} d+c^{4} d^{2}$.

For $m \equiv 2 \bmod 8$, let $m=8 u+2$ and $w_{2^{s}}(\xi)=c^{8 u+2} d^{2^{s-1}-4 u-1}$ with $2^{s-1}>4 u+1$. If $n>2^{s-1}-4 u-1$, then

$$
\mathrm{Sq}^{2} w_{2^{s}}(\xi)=c^{8 u+2} d^{2^{s-1}-4 u} \neq 0
$$

and so $2^{s} \leq 4$, giving $2 \geq 2^{s-1}>4 u+1$, so $u=0$ and $m=2$. For $m=2,2^{s}=4$ and $w_{4}(\xi)=c^{2} d$ one finds by Fujii and Yasui [3] that $\mathrm{KO}(P(2,1)) \leftarrow \mathrm{KO}(P(2,2))$ is epic $(2 r+1=1$ implies $r$ even $)$ and then $\mathrm{KO}(P(2,2)) \leftarrow \operatorname{KO}(P(2,2 r))$ is epic for all $r \geq 1$. Hence, there would be bundles for all $n \geq 1$. For $2^{s} \geq 8, w_{2^{s}}(\xi)=c^{8 u+2} d^{2^{s-1}-4 u-1}$ in $\mathrm{KO}\left(P\left(8 u+2,2^{s-1}-4 u-1\right)\right)$ has $2 r+1=2^{s-1}-4 u-1 \equiv 3 \bmod 4$ so $r$ is odd. By Fujii and Yasui [3] there is a stable bundle $\varrho^{\prime}$ in $\operatorname{KO}(P(8 u+2,2 r+1))$ for which $2 \varrho^{\prime}$ is in the image of $\mathrm{KO}(P(8 u+2,2 r+2))$, but $\varrho^{\prime}$ is not.

Thus one has

FACT. If $\xi$ is a vector bundle over $P(m, n)$ with $w(\xi)=1+w_{2^{s}}(\xi)+$ higher terms and not of the form $(1+c)^{a}(1+c+d)^{b}$, then:

(1) $w_{4}(\xi)=c^{2} d$ in $P(2, n)$ with $n \geq 1$,

(2) $w_{2^{s}}(\xi)=c^{8 u+2} d^{2^{s-1}-4 u-1}$ in $P\left(8 u+2,2^{s-1}-4 u-1\right) ; 2^{s} \geq 8$,

(3) $w_{8}(\xi)=c^{4} d^{2}$ in $P(4, n)$ or $P(5, n)$ with $n \geq 2$, or

(4) $w_{8}(\xi)= \begin{cases}c^{6} d & \text { in } P(6,1), \\ c^{6} d+c^{4} d^{2} & \text { in } P(6, n) \text { with } n \geq 2 .\end{cases}$

Now $P(2,1)$ and $P(6,1)$ have dimension 4 and 8 so admit degree one maps to $S^{4}$ and $S^{8}$. For $k=4$ and 8 , there is a vector bundle $\varrho$ over $S^{k}$ with $w_{k}(\varrho) \neq 0$. Pulling the bundle $\varrho$ back, one obtains vector bundles with

$$
w(\xi)=1+c^{2} d \quad \text { in } P(2,1) \text { and } \quad w(\xi)=1+c^{6} d \quad \text { in } P(6,1) .
$$

Using the epimorphisms in KO-theory as indicated above, the bundles for the cases (1) and (4) exist for all $n$ values. Restricting the bundles for $P(6, n)$, $n \geq 2$, to $P(4, n)$ and $P(5, n)$ gives the bundles of case (3) for all $n$.

Also, $P(2,3)$ has dimension 8 , and pulling back the bundle $\varrho$ from $S^{8}$ gives a bundle with

$$
w(\xi)=1+c^{2} d^{3} \quad \text { in } P(2,3),
$$

which is an example for case (2). All other possibilities in case (2) have $2^{s} \geq 16$, for $2^{s}=8>8 u+2$ forces $u=0$.

3. The classes. From the previous calculations one knows that for certain $P(m, n)$ there is a vector bundle $\xi$ over $P(m, n)$ for which $w(\xi)=$ 
$1+w_{2^{s}}(\xi)+$ higher terms, with $w_{2^{s}}(\xi)$ being known. One wants to know the complete class $w(\xi)$ for some such bundle.

For case $(2), P\left(8 u+2,2^{s-1}-4 u-1\right)$ with $2^{s} \geq 8$, it is clear that $w(\xi)=1+w_{2^{s}}(\xi)$ since the dimension of the manifold is $2^{s}$, and all higher terms must be zero.

The main tool is the $\mathrm{Wu}$ formula

$$
\begin{aligned}
\operatorname{Sq}^{i} w_{j} & =\sum_{t=0}^{i}\left(\begin{array}{c}
j-i-1+t \\
t
\end{array}\right) w_{i-t} w_{j+t} \quad \text { for } i<j \\
& =\left(\begin{array}{c}
j-1 \\
i
\end{array}\right) w_{i+j}+\text { decomposable terms }
\end{aligned}
$$

and in particular,

$$
\mathrm{Sq}^{i} w_{2^{t}}=\left(\begin{array}{c}
2^{t}-1 \\
i
\end{array}\right) w_{2^{t}+i}+\text { decomposables }=w_{2^{t}+i}+\text { decomposables. }
$$

First, consider $m=2$. Let $\xi$ be a bundle over $P(2, n)$ with $n$ large having $w(\xi)=1+c^{2} d+$ higher terms. Then

$$
\mathrm{Sq}\left(c^{2} d\right)=c^{2}(1+c)^{2} d(1+c+d)=c^{2} d(1+d)
$$

gives

$$
w(\xi)=1+c^{2} d+c^{2} d^{2}+\text { terms of dimension } \geq 8 .
$$

Inductively, suppose one has found $\xi$ with $w(\xi)=1+c^{2} d+c^{2} d^{2}+\ldots+c^{2} d^{r}+\ldots+c^{2} d^{2^{t}-2}+$ terms of dimension $\geq 2^{t+1}$, with $t \geq 2$. One then has $w_{2^{t+1}}(\xi)=\alpha c^{2} d^{2^{t}-1}+\beta d^{2^{t}}$ and if $\beta \neq 0$ one may add to $\xi$ a bundle with class

$$
\left(\frac{1+c+d}{1+c}\right)^{2^{t}}=1+d^{2^{t}}+\ldots
$$

to obtain a bundle with $\beta=0$, so may suppose $\beta=0$.

Letting $\xi^{\prime}$ be the restriction of $\xi$ to $P(1, n)$, one has $w_{i}\left(\xi^{\prime}\right)=0$ for $0<i \leq 2^{t+1}$ so $w_{i}\left(\xi^{\prime}\right)=0$ for $i<2^{t+2}$. Thus on $P(2, n)$ every class $w_{i}(\xi)$ is divisible by $c^{2}$ for $0<i<2^{t+2}$, and every product of two such classes is zero (i.e., decomposables are zero).

Then

$$
\operatorname{Sq}\left(w_{2^{t+1}}(\xi)\right)=w_{2^{t+1}}(\xi)+w_{2^{t+1}+1}(\xi)+\ldots+w_{2^{t+2}-1}(\xi)+w_{2^{t+2}}^{2}(\xi)
$$

is

$$
\begin{aligned}
\operatorname{Sq}\left(\alpha c^{2} d^{2^{t}-1}\right) & =\alpha c^{2} d^{2^{t}-1}(1+c+d)^{2^{t}-1} \\
& =\alpha c^{2} d^{2^{t}-1}(1+d)^{2^{t}-1}=\alpha\left\{c^{2} d^{2^{t}-1}+c^{2} d^{2^{t}}+\ldots+c^{2} d^{2^{t+1}-2}\right\} .
\end{aligned}
$$


Then

$$
\operatorname{Sq}^{4}\left(w_{2^{t+1}-2}(\xi)\right)=\left(\begin{array}{c}
2^{t+1}-3 \\
4
\end{array}\right) w_{2^{t+1}+2}=w_{2^{t+1}+2}
$$

is

$$
\mathrm{Sq}^{4}\left(c^{2} d^{2^{t}-2}\right)=c^{2} d^{2^{t}}
$$

giving $\alpha=1$. This completes the induction.

Hence, there is a $\xi$ over $P(2, n)$ with

$$
w(\xi)=1+c^{2} d+c^{2} d^{2}+\ldots+c^{2} d^{r}+\ldots=1+\frac{c^{2} d}{1+d}=1+\frac{c^{2} d}{1+c+d} .
$$

Then

$$
\begin{aligned}
w(\xi) \cdot(1+c)^{2}(1+c+d) & =\left(1+\frac{c^{2} d}{1+c+d}\right)\left(1+c^{2}\right)(1+c+d) \\
& =\left(1+c^{2}+\frac{c^{2} d}{1+c+d}\right)(1+c+d) \\
& =\left(1+\frac{c^{2}}{1+c+d}\right)(1+c+d) \\
& =1+c+\left(d+c^{2}\right),
\end{aligned}
$$

giving a bundle with Stiefel-Whitney class $1+c+\left(d+c^{2}\right)$, as in the proposition.

Comment. There is a 2-plane bundle over $P(2, \infty)$ with $w(\xi)=1+$ $c+\left(d+c^{2}\right)$. For two 2-plane bundles $\xi_{1}$ and $\xi_{2}$ over the space $X$ with the same first Stiefel-Whitney class, i.e., with $w\left(\xi_{i}\right)=1+x+w_{2}\left(\xi_{i}\right)$, there is a tensor product which is again a 2-plane bundle with $w\left(\xi_{1} \otimes \xi_{2}\right)=$ $1+x+\left(w_{2}\left(\xi_{1}\right)+w_{2}\left(\xi_{2}\right)\right)$. This bundle can be obtained by a map

$$
\frac{S^{\infty} \times \mathbb{C} P^{\infty} \times \mathbb{C} P^{\infty}}{-1 \times(\text { conj }) \times(\text { conj })} \rightarrow \frac{S^{\infty} \times \mathbb{C} P^{\infty}}{-1 \times(\text { conj })}
$$

where conj $=$ conjugation and the map from $\mathbb{C} P^{\infty} \times \mathbb{C} P^{\infty}$ to $\mathbb{C} P^{\infty}$ sends $\left(\left[z_{i}\right],\left[w_{j}\right]\right)$ to the point with homogeneous coordinates $\left[z_{i} w_{j}\right]$, or equivalently from a homomorphism from

$$
\left\{\left(g_{1}, g_{2}\right) \in O_{2} \times O_{2} \mid \operatorname{det} g_{1}=\operatorname{det} g_{2}\right\} \rightarrow O_{2} .
$$

The tensor product of the tangent bundle of $\mathbb{R} P^{2}$ pulled back to $P(2, n)$ has $w(\tau)=(1+c)^{3}=1+c+c^{2}$ and the bundle $\eta$ has $w(\eta)=1+c+d$, so $w(\tau \otimes \eta)=1+c+\left(d+c^{2}\right)$.

For $m=4$ or 5 the argument is almost identical with the argument for $m=2$. If $\xi$ is a bundle over $P(m, n)$ with $n$ large having $w(\xi)=1+c^{4} d^{2}+$ higher terms, then

$$
w(\xi)=1+c^{4} d^{2}+c^{4} d^{4}+\text { terms of dimension } \geq 16 .
$$


Inductively, suppose one has found $\xi$ with $w(\xi)=1+c^{4} d^{2}+c^{4} d^{4}+\ldots+c^{4} d^{2}+\ldots+c^{4} d^{2^{t}-4}+$ terms of dimension $\geq 2^{t+1}$ and $t \geq 3$. One then has $w_{2^{t+1}}(\xi)=\alpha c^{4} d^{2^{t}-2}+\beta c^{2} d^{2^{t}-1}+\gamma d^{2^{t}}$, and by adding a bundle with class

$$
\left(\frac{1+c+d}{1+c}\right)^{2^{t}}=1+d^{2^{t}}+\ldots
$$

may suppose $\gamma=0$.

If $\xi^{\prime}$ is the restriction of $\xi$ to $P(3, n)$ one has $w\left(\xi^{\prime}\right)=1+\beta c^{2} d^{2^{t}-1}+\ldots$ and hence $\beta=0$, for there is no bundle over $P(3, n)$ having this form with $\beta \neq 0$. Then $w_{i}\left(\xi^{\prime}\right)=0$ for $0<i<2^{t+2}$ and every class $w_{i}(\xi)$ is divisible by $c^{4}$ for $0<i<2^{t+2}$. The product of any two such classes is then zero.

Beyond this point, the argument is identical with the case $m=2$, and one obtains a bundle $\xi$ with $w(\xi)=\left(1+c+\left(d+c^{2}\right)\right)^{2}$.

Now consider $m=6$ and let $\xi$ be a bundle over $P(6, n)$ with $n$ large having $w(\xi)=1+\left(c^{6} d+c^{4} d^{2}\right)+$ higher terms. If $\xi^{\prime}$ is the restriction of $\xi$ to $P(5, n)$, then $w\left(\xi^{\prime}\right)=1+c^{4} d^{2}+\ldots$, and multiplying by a power of $((1+c+d)(1+c))$, one may suppose that $w\left(\xi^{\prime}\right)=1+c^{4} d^{2}+c^{4} d^{4}+\ldots+$ $c^{4} d^{2 r}+\ldots$

Thus

$$
w(\xi)=1+\left(c^{6} d+c^{4} d^{2}\right)+c^{4} d^{4}+c^{4} d^{6}+\ldots+\text { terms divisible by } c^{6},
$$

and all decomposable classes are zero.

Now

$$
\begin{aligned}
\mathrm{Sq}\left(w_{8}(\xi)\right) & =\operatorname{Sq}\left(c^{6} d+c^{4} d^{2}\right)=c^{6} d(1+c+d)+c^{4}(1+c)^{4} d^{2}(1+c+d)^{2} \\
& =c^{6} d(1+d)+c^{4} d^{2}\left(1+c^{2}+d^{2}\right)=\left(c^{6} d+c^{4} d^{2}\right)+c^{4} d^{4},
\end{aligned}
$$

and

$$
w(\xi)=1+\left(c^{6} d+c^{4} d^{2}\right)+c^{4} d^{4}+\left(\alpha c^{6} d^{5}+c^{4} d^{6}\right)+\text { higher terms. }
$$

Inductively, one assumes

$$
w(\xi)=1+\sum c^{6} d^{4 i+1}+\sum c^{4} d^{2 j}+\underset{w_{2^{t+1}}(\xi)}{\left(\alpha c^{6} d^{2^{t}-3}+c^{4} d^{2^{t}-2}\right)+\text { higher terms }}
$$

where the initial sums are the terms of dimension less than $2^{t+1}$, and $t \geq 3$.

Then $w_{2^{t+1}-2}(\xi)=c^{4} d^{2^{t}-4}$ and

$$
\begin{aligned}
w_{2^{t+1}+2}(\xi) & =\left(\begin{array}{c}
2^{t+1}-3 \\
4
\end{array}\right) w_{2^{t+1}+2}(\xi)=\operatorname{Sq}^{4}\left(w_{2^{t+1}-2}(\xi)\right) \\
& =\operatorname{Sq}^{4}\left(c^{4} d^{2^{t}-4}\right)=c^{4} \mathrm{Sq}^{4} d^{2^{t}-4}=c^{4}\left(c^{4} d^{2^{t}-4}\right)=0,
\end{aligned}
$$


with

$$
\begin{aligned}
w_{2^{t+1}+2}(\xi) & =\operatorname{Sq}^{2}\left(w_{2^{t+1}}(\xi)\right)=\mathrm{Sq}^{2}\left(\alpha c^{6} d^{2^{t}-3}+c^{4} d^{2^{t}-2}\right) \\
& =\alpha c^{6} \mathrm{Sq}^{2} d^{2^{t}-3}+c^{4} \mathrm{Sq}^{2} d^{2^{t}-2}=\alpha c^{6} d^{2^{t}-2}+c^{4}\left(c^{2} d^{2^{t}-2}\right) \\
& =(\alpha+1) c^{6} d^{2^{t}-2},
\end{aligned}
$$

so $\alpha=1$.

Then

$$
\begin{aligned}
\mathrm{Sq}\left(w_{2^{t+1}}(\xi)\right) & =\mathrm{Sq}\left(c^{6} d^{2^{t}-3}+c^{4} d^{2^{t}-2}\right) \\
& =c^{6} d^{2^{t}-3}(1+d)^{2^{t}-3}+c^{4}(1+c)^{4} d^{2^{t}-2}(1+c+d)^{2^{t}-2} \\
& =c^{6} d^{2^{t}-3}(1+d)(1+d)^{2^{t}-4}+c^{4} d^{2^{t}-2}\left(1+c^{2}+d^{2}\right)(1+d)^{2^{t}-4} \\
& =\left\{c^{6} d^{2^{t}-3}+c^{6} d^{2^{t}-2}+c^{4} d^{2^{t}-2}+c^{6} d^{2^{t}-2}+c^{4} d^{2^{t}}\right\}(1+d)^{2^{t}-4} \\
& =c^{6} d^{2^{t}-4+1}(1+d)^{2^{t}-4}+c^{4} d^{2^{t}-2}(1+d)^{2^{t}-4}
\end{aligned}
$$

completing the induction up to the next power of 2 .

Thus

$$
w(\xi)=1+\frac{c^{6} d}{(1+d)^{4}}+\frac{c^{4} d^{2}}{(1+d)^{2}}
$$

Now

$$
w(\xi) \cdot(1+c)^{4}=1+c^{4}+\frac{c^{6} d}{(1+d)^{4}}+\frac{c^{4} d^{2}}{(1+d)^{2}}=1+\frac{c^{4}}{(1+d)^{2}}+\frac{c^{6} d}{(1+d)^{4}},
$$

and

$$
\begin{aligned}
w(\xi)(1+c)^{4}(1+c+d)^{2}= & (1+c+d)^{2} \\
& +\frac{c^{4}}{(1+d)^{2}}\left\{(1+d)^{2}+c^{2}\right\}+\frac{c^{6} d(1+d)^{2}}{(1+d)^{4}} \\
= & (1+c+d)^{2}+c^{4}+\frac{c^{6}}{(1+d)^{2}}+\frac{c^{6} d}{(1+d)^{2}} \\
= & \left(1+c+\left(d+c^{2}\right)\right)^{2}+\frac{c^{6}}{1+d},
\end{aligned}
$$

and

$$
w(\xi)(1+c)^{4}(1+c+d)^{3}=\left(1+c+\left(d+c^{2}\right)\right)(1+c+d)+c^{6},
$$

giving the existence of a bundle with class $\left(1+c+\left(d+c^{2}\right)\right)^{2}(1+c+d)+c^{6}$, as in the proposition.

Comment. The classes of the unusual bundles over $P(4, n)$ and $P(5, n)$ or $P(6, n)$ look like the classes of 4 -plane or 6 -plane bundles. It would be interesting to know if these are the classes of such bundles. 
4. The nonbundles. To complete the proof of the Proposition, one needs to show that there can be no bundle over $P\left(8 u+2,2^{s-1}-4 u-1\right)$ with $2^{s} \geq 16$ for which $w(\xi)=1+c^{8 u+2} d^{2^{s-1}-4 u-1}$.

Letting $m=8 u+2$ and $n=2^{s-1}-4 u-1$ with $2^{s} \geq 16$, Fujii and Yasui [3] show that the homomorphism

$$
\mathrm{KO}(P(m, n)) \leftarrow \mathrm{KO}(P(m, n+1)) \oplus \mathrm{KO}(P(m, n) / P(m, n-1))
$$

is epic. From our calculations every vector bundle over $P(m, n+1)$ has Stiefel-Whitney class of the form

$$
\begin{cases}(1+c)^{a}(1+c+d)^{b} & \text { if } u>0 \\ (1+c)^{a}(1+c+d)^{b}\left(1+c+\left(d+c^{2}\right)\right)^{\varepsilon} & \text { if } u=0 ; \varepsilon=0 \text { or } 1 .\end{cases}
$$

To complete the proof, it then suffices to show

FACT. For every vector bundle $\varrho$ over $P(m, n) / P(m, n-1)$ one has $w(\varrho)=1$.

The space $P(m, n) / P(m, n-1)$ can be described in several ways. On $P(m, n)=S^{m} \times \mathbb{C} P^{n} /(-1 \times$ conjugation $)$ one has the involution induced by $1 \times T$ where

$$
T\left(\left[z_{0}, z_{1}, \ldots, z_{n}\right]\right)=\left[-z_{0}, z_{1}, \ldots, z_{n}\right] .
$$

This involution fixes $\mathbb{R} P^{m}=S^{m} \times \mathbb{C} P^{0} /(-1 \times$ conjugation $)$ and $P(m, n-1)$, and $P(m, n) / P(m, n-1)=T(\nu)$ is the Thom space of the normal bundle of $\mathbb{R} P^{m}$ in $P(m, n)$. The normal bundle of $\mathbb{C} P^{0}$ in $\mathbb{C} P^{n}$ is $\mathbb{C}^{n}$ with the involution conjugation, so the normal bundle of $\mathbb{R} P^{m}$ in $P(m, n)$ is $\left(S^{m} \times \mathbb{C}^{n}\right) /(-1 \times$ conjugation $)$ and $\nu=n \ell+n$ where $\ell$ is the nontrivial line bundle over $\mathbb{R} P^{m}$. Thus $T(\nu)=T(n \ell+n)=\Sigma^{n} T(n \ell)$ is the $n$-fold suspension of the Thom space of $n \ell$ over $\mathbb{R} P^{m}$. On $\mathbb{R} P^{m+n}$, one has the linear involution which is -1 in $m+1$ variables that fixes $\mathbb{R} P^{m}$, with normal bundle $n \ell$, and $\mathbb{R} P^{n-1}$, with normal bundle $m \ell$, and $\mathbb{R} P^{m+n} / \mathbb{R} P^{n-1}=T(n \ell)$ is the Thom space of the normal bundle of $\mathbb{R} P^{m}$ in $\mathbb{R} P^{m+n}$. Thus

$$
\frac{P(m, n)}{P(m, n-1)}=\Sigma^{n} T(n \ell)=\Sigma^{n}\left(\frac{\mathbb{R} P^{m+n}}{\mathbb{R} P^{n-1}}\right) .
$$

Now, Atiyah and Hirzebruch [1] show that every vector bundle over a 9 -fold suspension has trivial Stiefel-Whitney class; i.e., $w(\varrho)=1$ for $n \geq 9$.

Since $n=2^{s-1}-4 u-1>0$ is congruent to $3 \bmod 4$, one is reduced to considering $n=3$ and $n=7$.

For the inclusion

$$
\Sigma^{n}\left(\frac{\mathbb{R} P^{m-1+n}}{\mathbb{R} P^{n-1}}\right) \rightarrow \Sigma^{n}\left(\frac{\mathbb{R} P^{m+n}}{\mathbb{R} P^{n-1}}\right),
$$


with $m=8 u+2$ and $n=2 r+1$, with $r$ odd, Fujii and Yasui [3] give

$$
\widetilde{\mathrm{KO}}\left(\Sigma^{n}\left(\frac{\mathbb{R} P^{m-1+n}}{\mathbb{R} P^{n-1}}\right)\right) \leftarrow \widetilde{\mathrm{KO}}\left(\Sigma^{n}\left(\frac{\mathbb{R} P^{m+n}}{\mathbb{R} P^{n-1}}\right)\right)
$$

from which it follows that every bundle over $\Sigma^{n}\left(\mathbb{R} P^{m+n} / \mathbb{R} P^{n-1}\right)$ actually comes from

$$
\Sigma^{n}\left(\frac{\mathbb{R} P^{m+n}}{\mathbb{R} P^{m+n-1}}\right)=\Sigma^{n}\left(S^{m+n}\right)=S^{m+2 n}=S^{2^{s}} .
$$

Since every bundle over $S^{2^{s}}$ with $2^{s} \geq 16$ has trivial Stiefel-Whitney class (again [1]), one obtains $w(\varrho)=1$ as was asserted.

\section{References}

[1] M. F. Atiyah and F. Hirzebruch, Bott periodicity and the parallelizability of the spheres, Proc. Cambridge Philos. Soc. 57 (1961), 223-226.

[2] A. Dold, Erzeugende du Thomschen Algebra N, Math. Z. 65 (1956), 25-35.

[3] M. Fujii and T. Yasui, $K_{0}$-cohomologies of the Dold manifolds, Math. J. Okayama Univ. 16 (1973), 55-84.

[4] J. J. Ucci, Immersions and embeddings of Dold manifolds, Topology 4 (1965), 283-293.

Department of Mathematics

University of Virginia

P.O. Box 400137

Charlottesville, VA 22904-4137, U.S.A.

Received 18 September 2000;

in revised form 20 December 2000 